\title{
Simplified Harmonic Rejection Mixer Analysis and Design Based on a Filtered Periodic Impulse Model
}

\author{
Pieter-Tjerk de Boer, Mark S. Oude Alink, Senior Member, IEEE, Eric A.M. Klumperink, Fellow, IEEE
}

\begin{abstract}
Hard-switched mixers have attractive linearity and noise properties, but are plagued by spurious responses due to the harmonic content of square-wave local oscillator (LO) signals. Harmonic rejection mixers (HRMs) reject (some of) these responses and can be constructed combining polyphase mixing and amplitude weighting. This paper presents a generalized model for the analysis of the harmonic rejection (HR) of such HRMs, based on circular convolution. We show that the effective LO signal can be modeled as a periodic Dirac impulse filtered by a time-discrete filter. For a multi-stage HR system, this filter consists of multiple stages as well, and the coefficients of each stage can be found simply by inspection. The total HR is shown to be the sum (in $\mathrm{dB}$ ) of the rejection of the individual filtering stages, highly simplifying analysis and design of HRMs.
\end{abstract}

Index Terms-circular convolution, frequency conversion, harmonic rejection, mixer

\section{INTRODUCTION}

$\mathbf{H}$ ARD-SWITCHED PASSIVE MIXERS are commonly used in radio receivers, because they can achieve excellent linearity and noise, resulting in high spurious-free dynamic range, i.e. good interference robustness [1]-[3]. However, the square-wave local oscillator (LO) waveform contains strong harmonic components, which cause interferers at these harmonic frequencies to be downconverted to the same intermediate frequency (IF). In transmitters, harmonic mixing produces unwanted transmitted signal components at harmonics of the fundamental LO frequency. In both cases harmonic rejection mixers (HRMs), which suppress the response at harmonics of the LO frequency, are highly desired. For a tutorial on harmonic rejection mixers we refer to [4].

In the scientific literature, [5] is often (implicitly or explicitly) cited as the earliest example of a HRM [1], [6], [7]. The idea of using switching mixers with appropriate sampled sinewave gains to reduce harmonics, however, is at least 13 years older [8], see Fig. 1. Since then, many different techniques have been proposed to implement HRMs, including for example [1], [6], [7], [9], [10].

The design of HRMs is often discussed intuitively as periodically switching between different gain values. The gain as a piecewise-constant function of time is then viewed as an "effective LO waveform" lacking certain harmonics. In the

Accepted for TCAS-II, January 2021

P.T. de Boer is with the Design and Analysis of Communication Systems group, Faculty of Electrical Engineering, Mathematics and Computer Science (EEMCS), University of Twente, 7500 AE Enschede, The Netherlands.

M.S. Oude Alink and E.A.M. Klumperink are with the IC-Design Group, EEMCS, University of Twente, 7500 AE Enschede, The Netherlands.

Copyright (c) 2020 IEEE. Personal use of this material is permitted. However, permission to use this material for any other purposes must be obtained from the IEEE by sending an email to pubs-permissions@ieee.org.

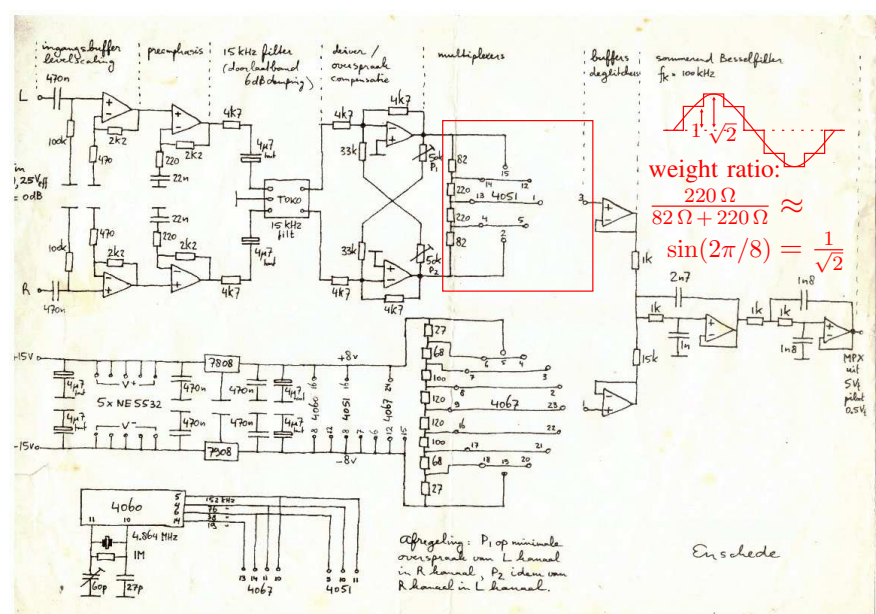

Fig. 1. Annotated photo of FM stereo encoder schematic for a Dutch university campus radio station from circa 1988 [8], containing what may be the first HRM. The 4051 and 4067 are CMOS analog multiplexer chips, and the resistor networks determine the various gains.

case of Fig. 1, a CMOS switch switches periodically between various resistors to achieve gain ratios of $1: \sqrt{2}: 1$ at $\frac{2 \pi}{8}$ phase increments. It can easily be verified by Fourier analysis that the $2^{\text {nd }}$ through $6^{\text {th }}$ harmonics are canceled in this effective LO-waveform. The same weights $1: \sqrt{2}: 1$ are commonly used in 8-phase receiver mixers in various forms [11]-[13].

Using an arbitrary number of $n$ paths and taking $n$ equidistant samples of a sinewave as weights, up to the $(n-1)$ th harmonic can be suppressed [6], [7]. In a straightforward implementation, $30-40 \mathrm{~dB}$ of harmonic rejection (HR) is typically achieved, limited by inaccuracies in phase (approx. $1^{\circ}$ ) and amplitude (approx. 1\%) due to device mismatch. Higher HR can be achieved by device scaling or tuning [11], [13]. Here we focus on HR improvement by circuit topology changes, namely moving the matching requirements to baseband ([6], > $50 \mathrm{~dB} \mathrm{HR}$ ), using 2-stage weighting ([1], $>60 \mathrm{~dB} \mathrm{HR}$ ), or a combination of the two ([7], > $70 \mathrm{~dB}$ ). Even higher HR may be achieved by e.g. adaptive calibration and the use of tracking filters, see e.g. [3], [9], but those are outside the scope of this article.

It is non-trival to implement non-integer ratios like $1: \sqrt{2}$ directly [5], [9], [11]-[13]. Integer ratios such as 2:3 [1], 5:7 [1], 11:16 [2], 12:17 [10] or sometimes even higher [6], [7] are used to enable the use of matched unit elements. Although many sets of weighting coefficients are possible, it is not obvious how to evaluate the achievable HR and optimize it in multi-stage designs. This paper targets the analysis of the HR 


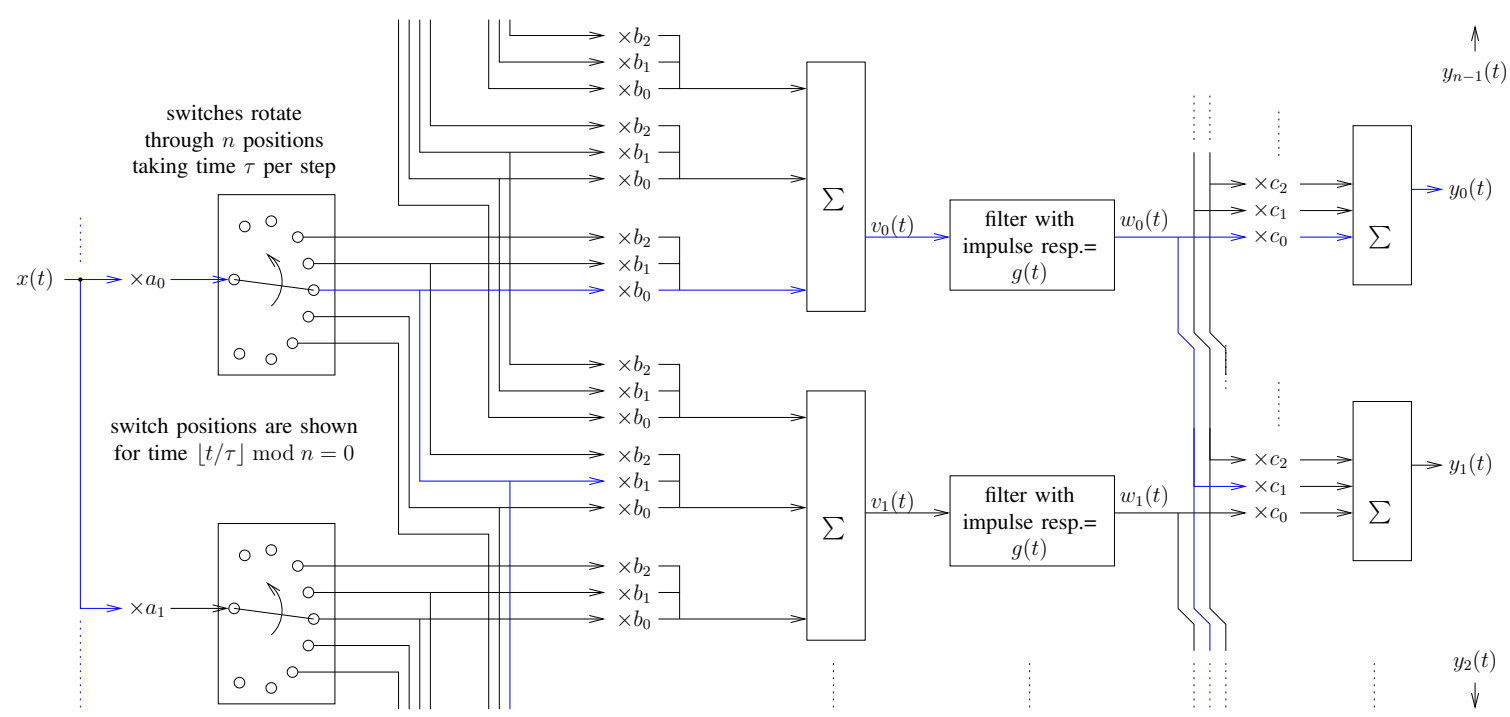

Fig. 2. Schematic description of our mixer model, with $x(t)$ the input signal, and $y_{m}(t)$ the output signals. Note that the structure sketched here twice, is conceptually present not two but $n$ times, with the top and bottom of the diagram circularly connected (i.e., indices taken modulo $n$ ). Note further that for simplicity the figure is drawn as if the vectors $\boldsymbol{b}$ and $\boldsymbol{c}$ have only three non-zero elements, but the generalization up to $b_{n-1}$ and $c_{n-1}$ is straightforward. The blue color has no special meaning other than highlighting the structure.

in polyphase mixers with multi-stage weighting coefficients.

We propose a generalized block diagram and derive the HR based on cyclic convolution. This leads to a new way to find the effective LO waveform as a periodic sequence of Dirac pulses filtered by a multi-stage time-discrete filter. It also provides design insights for selecting the weight ratios.

The paper is structured as follows: Section II introduces and analyzes a generalized polyphase mixer model with three stages of weighting coefficients, leading to the new effective LO waveform model. Section III applies the model to some key HRM publications. Section IV ends with conclusions.

\section{ANALYSIS}

\section{A. Generalized Polyphase Mixing and Weighting Model}

Consider a polyphase passive mixer, modeled in Fig. 2 as a rotating switch to stress periodic behavior. The switch has $n$ positions, and stays a time $\tau$ in each position (equal on-time), so complete rotations happen at frequency $1 / n \tau$. Assume the switches are connected to their environment by three sets of "weighting coefficients": 1) before the mixing switches, to weight the inputs; 2) between the switches and a filter that selects the wanted output band (e.g. low-pass, in case of a zero-IF down-mixer and band-pass for a transmitter, or no filter at all in some cases); 3) after the filters to produce a weighted output. We assume unilateral weighting blocks for simplicity. The filters are assumed to have the same (potentially infinitely long, but causal) impulse response $g(t)$.

The three sets of weighting coefficients are denoted by $a$, $\boldsymbol{b}$ and $\boldsymbol{c}$, each of which is a vector whose $i$ th component is denoted $a_{i}, b_{i}$ and $c_{i}$, respectively. For an $n$-phase system, the switches have $n$ positions, so in general there can be $n$ branches of filters and outputs, two of which are shown in the figure. For notational convenience, we periodically extend the coefficients as $a_{i}=a_{i \bmod n}$ and similarly for $\boldsymbol{b}$ and $\boldsymbol{c}$.

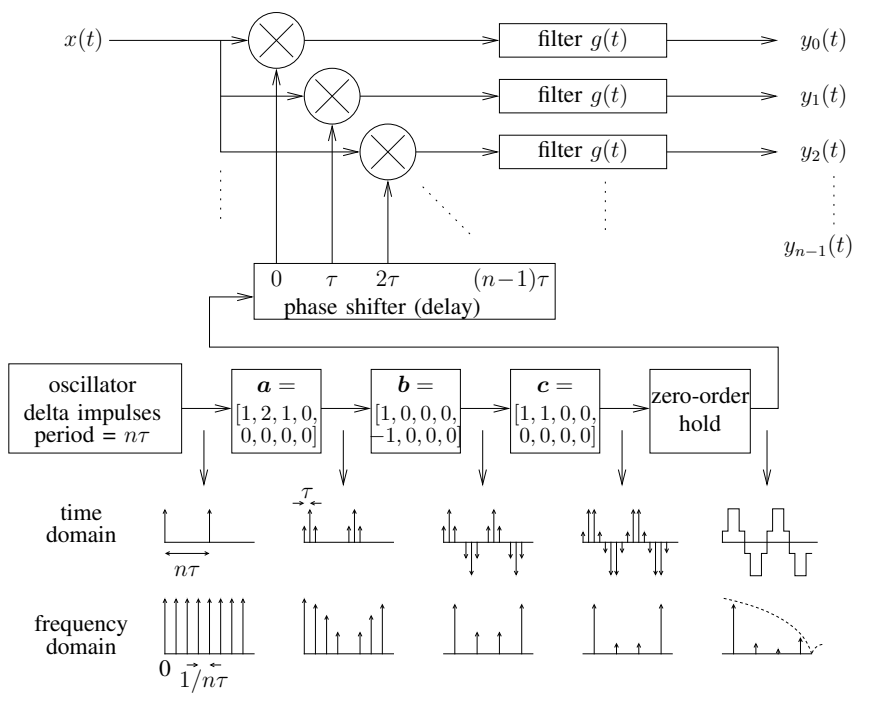

Fig. 3. Equivalent view of the model, showing filtering in the LO path. The values for $\boldsymbol{a}, \boldsymbol{b}$ and $\boldsymbol{c}$ and the sketches of the signals are an illustrative example for $n=8$ : here $\boldsymbol{b}$ suppresses all even harmonics, $\boldsymbol{a}$ also suppresses the $4^{\text {th }}$ harmonic, and $\boldsymbol{a}$ and $\boldsymbol{c}$ both attenuate the $3^{\text {rd }}$ and $5^{\text {th }}$ harmonics.

The connections in the figure may look chaotic at first, but they are quite systematic. Whenever a signal goes from branch $i$ to branch $i+k$ (modulo $n$ ), the multiplicative coefficient of this contribution is an element of the vector $\boldsymbol{a}$, $\boldsymbol{b}$ or $\boldsymbol{c}$, whose index is found by adding $k$ to the index of the contribution staying inside branch $i$. Note that this connection pattern matches the result derived in Appendix I.B of [14].

\section{B. Convolving Coefficient Sets}

We consider signals in continuous time, with timestep $\tau$ being the time for one step of the switches. Denote the input signal at time $t$ as $x(t)$, and the output of the $m$ th branch as $y_{m}(t)$. Intermediate signals are denoted by $v_{m}(t)$ and 
$w_{m}(t)$ as indicated in Fig. 2. Similar to the subscripts of $\boldsymbol{a}$, $\boldsymbol{b}$ and $\boldsymbol{c}$, the subscript of $v_{m}(t)$ and $w_{m}(t)$ is also assumed periodic, i.e., taken modulo $n$.

By inspection we find from Fig. 2 that:

$$
\begin{aligned}
y_{m}(t) & =\sum_{j=0}^{n-1} c_{m-j} w_{j}(t), \\
w_{j}(t) & =\int_{0}^{\infty} g(u) v_{j}(t-u) \mathrm{d} u, \\
v_{j}(t) & =\sum_{\ell=0}^{n-1} b_{\left\lfloor\frac{t}{\tau}\right\rfloor+j-\ell} a_{\ell} x(t) .
\end{aligned}
$$

Taking this all together gives:

$$
\begin{aligned}
y_{m}(t) & =\sum_{j=0}^{n-1} c_{m-j} \int_{0}^{\infty} g(u) \sum_{\ell=0}^{n-1} b_{\left\lfloor\frac{t-u}{\tau}\right\rfloor+j-\ell} a_{\ell} x(t-u) \mathrm{d} u \\
& =\int_{0}^{\infty} g(u) \sum_{j^{\prime}=0}^{n-1} c_{m-j^{\prime}+\left\lfloor\frac{t-u}{\tau}\right\rfloor} \sum_{\ell=0}^{n-1} b_{j^{\prime}-\ell} a_{\ell} x(t-u) \mathrm{d} u \\
& =\int_{0}^{\infty} g(u) e_{\left\lfloor\frac{t-u}{\tau}\right\rfloor+m} x(t-u) \mathrm{d} u
\end{aligned}
$$

with

$$
e_{i}=\sum_{j=0}^{n-1} \sum_{\ell=0}^{n-1} c_{i-j} b_{j-\ell} a_{\ell} .
$$

Note that $e$ is simply the (circular) convolution of $\boldsymbol{a}, \boldsymbol{b}$ and $\boldsymbol{c}$, which is again periodic with the same period $n$.

\section{Effective LO Construction}

We can take $e$ and $x(t)$ together in (4), resulting in:

$$
y_{m}(t)=\int_{0}^{\infty} g(u) z_{m}(t-u) \mathrm{d} u
$$

where

$$
z_{m}(t)=e_{\left\lfloor\frac{t}{\tau}\right\rfloor+m} x(t) .
$$

As $\boldsymbol{e}$ has period $n$, the input signal $x(t)$ is multiplied by a periodic signal with period $n \tau$, henceforth called the 'effective LO', to give $z_{m}(t)$, which in turn is filtered by convolution with $g(\cdot)$ to give the final output signal. The effective LO has, via the index $m$, a different phase for each output $y_{m}$.

The effective LO signal feeding the mixer is constructed as follows. Start with delta impulses, spaced $n \tau$ in time; this contains all harmonics of $1 / n \tau$ with equal amplitude. These delta impulses are filtered using circular convolution with vectors $\boldsymbol{a}, \boldsymbol{b}, \boldsymbol{c}$, in discrete time with time steps $\tau$. This results in the (periodic) vector $e$ representing the LO signal as a series of delta impulses, now spaced by $\tau$, and still with period $n \tau$. Such a signal has a spectrum containing only the discrete frequencies $\left\{0, \frac{1}{n \tau}, \frac{2}{n \tau}, \ldots, \frac{n-1}{n \tau}\right\}$, repeated every $1 / \tau$ to infinity. Thus, up to the $n-1^{\text {th }}$ harmonic can potentially be suppressed by choosing $\boldsymbol{a}, \boldsymbol{b}$ and/or $\boldsymbol{c}$ suitably. In fact, $\boldsymbol{a}$, $\boldsymbol{b}$ and $\boldsymbol{c}$ form three cascaded filters with a total (discretetime) impulse response $e$, as shown in Fig. 3. Finally, these $\boldsymbol{e}$-weighted delta impulses are convolved with a rectangle of duration $\tau$. This makes the signal piecewise continuous and acts as a low-pass filter, with a sinc-shaped frequency response having the first null at frequency $1 / \tau$. Clearly, the harmonic response (or rejection) of this mixer is purely determined by the harmonic content of the effective LO signal, which immediately follows from the responses of the cascaded filters.

Thus, the system acts as an ideal mixer/multiplier with a specific effective LO waveform, followed by a filter $g(\cdot)$. For a receiver, $g(\cdot)$ has no influence on the system HR, as the filter is applied after the harmonic responses fold on top of the desired signal. For a transmitter, however, $g(\cdot)$ can still improve the overall HR of the system.

The model can easily be extended with more linear combination stages at the right, which act as additional cascaded filters for the LO. And although the filter $g(\cdot)$ is now drawn between the $\boldsymbol{b}$ and $\boldsymbol{c}$ stages, such filters can be inserted anywhere to the right of the switches; e.g. between the switches and the $\boldsymbol{b}$ multipliers as in the example of Fig. $4 \mathrm{~b}$. Then $g(\cdot)$ is the convolution of the individual filters.

\section{Differential and quadrature signals}

In many practical designs, differential and/or I/Q outputs are desired. If both input and output are differential, no extra modelling is needed by treating all signals in the model as differential. Quadrature outputs are trivially realized if $n$ is a multiple of 4: if $y_{0}$ is the in-phase output, then $y_{n / 4}$ is the quadrature output. This can be seen by inspection, as the circuitry producing for example $y_{n / 4}$ is the same as for $y_{0}$, except that switch positions are used which are leading by a quarter period (i.e., $n / 4$ positions). Equivalently, (7) shows that $y_{n / 4}(t)$, via $z_{n / 4}(t)$, has its effective LO advanced by $n / 4$ timeslots compared to $y_{0}(t)$. Hence, an I/Q-output is obtained by implementing $y_{0}(t)$ and $y_{n / 4}(t)$. Similarly, for a single-ended input, a differential output is obtained by implementing both $y_{0}(t)$ and $y_{n / 2}(t)$, and a differential I/Qoutput by implementing $y_{0}(t), y_{n / 4}(t), y_{n / 2}(t)$ and $y_{3 n / 4}(t)$. Another option, as exploited in [7], see Fig. 4c, is to create phase-shifted copies of $c$, each having its own output.

A quadrature input for upconverting mixers, or even a complete set of $n$ phase-shifted inputs $x_{0}(t) \ldots x_{n-1}(t)$ may be desired, as explored in [14]. Our model can trivially be extended to this case: the input to the first switch would not just be $a_{0} x(t)$ as in Fig. 2, but a summation $\sum_{j=0}^{n-1} a_{j} x_{-j}(t)$, with the usual convention of indices modulo $n$. This leads to replacing (7) by $z_{m}(t)=\sum_{j=0}^{n-1} e_{\left\lfloor\frac{t}{\tau}\right\rfloor+m+j} x_{-j}(t)$ revealing indeed a $90^{\circ}$ phase difference between the effective LOs seen by the $x_{n}(t)$ and $x_{n / 4}(t)$ inputs.

\section{E. Consequences and insights}

From the obtained equivalent model, we can draw a few immediate conclusions about choosing $\boldsymbol{a}, \boldsymbol{b}$ and $\boldsymbol{c}$ (and any potential further stages):

- The coefficient sets $\boldsymbol{a}, \boldsymbol{b}, \boldsymbol{c}$ effectively describe independent cascaded filters. There is no need to search for a magical combination, as e.g. in [1]; one needs $\boldsymbol{a}, \boldsymbol{b}$ and/or $c$ to individually attenuate undesired harmonics.

- The filters effectively filter the LO, and therefore the width of their notches is unimportant, which is quite 


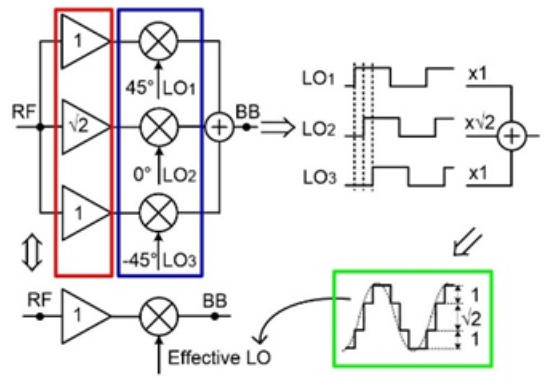

(a)

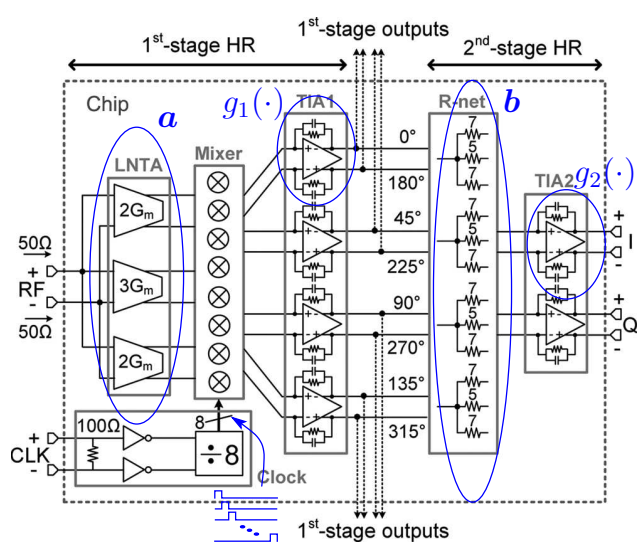

(b)

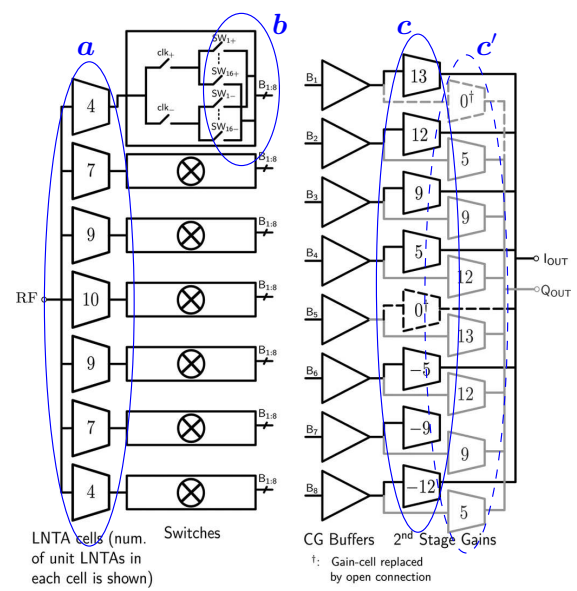

(c)

Fig. 4. (a) Weldon's HR transmitter [5] with 3 signal paths using $45^{\circ}$-shifted $50 \%$ duty-cycle square-waves and weighted 1 : $\sqrt{2}: 1$. Adapted from [3] (b) Annotated block schematic of Ru [1]. Note that $g(\cdot)$ is the convolution of the two parts $g_{1}(\cdot)$ and $g_{2}(\cdot)$, as discussed at the end of Section II-C (c) Annotated block schematic of Forbes [7]. Note that the Q output has been realized here by phase shifting $\boldsymbol{c}$ by 90 degrees to give $\boldsymbol{c}^{\prime}$.

different from designing a filter in the signal path itself As the filter coefficients $\boldsymbol{a}, \boldsymbol{b}$ and $\boldsymbol{c}$ are periodic, they only have a meaningful passband at discrete frequencies (namely the LO and its harmonics).

- It is possible to get HR by having cross-terms only in the baseband circuitry; i.e., choose $\boldsymbol{a}=\boldsymbol{b}=[1,0, \ldots 0]$ and rely solely on $c$ for HR (as done e.g. in [2]).

- As the filters are cascaded, the damage from manufacturing tolerances is reduced. E.g., if due to manufacturing tolerances the coefficients are not precise enough for more than $20 \mathrm{~dB}$ of harmonic suppression in each stage, the total suppression with $\boldsymbol{a}, \boldsymbol{b}, \boldsymbol{c}$ can still reach $60 \mathrm{~dB}$.

\section{EXAMPLES}

We now model several key HRMs to provide a representative illustration of the capabilities of our framework. Most other works are, from a modeling perspective, relatively straightforward deviations from the ones listed here.

\section{A. Jeffrey Weldon et al.}

The upconverting HRM in [5] consists of three currentcommutating submixers, see Fig. 4a. Each submixer is effectively a switch, operated with a $50 \%$ duty cycle LO, but with phases differing by $1 / 8$ period $\left(45^{\circ}\right)$. We can model this with $n=8$ and $\boldsymbol{a}=[1, \sqrt{2}, 1,0,0,0,0,0]$, and $\boldsymbol{b}=[1,1,1,1,-1,-1,-1,-1]$. Here $\boldsymbol{a}$ represents the three switches with different current sources setting their gains to 1 and $\sqrt{2}$, and each switch has a $50 \%$ duty cycle represented by the four $1 \mathrm{~s}$ in $\boldsymbol{b}$. The latter two stages in our model are absent in this mixer, represented by $g(t)=\delta(t)$, and eliminating all cross-connects in $\boldsymbol{c}$ as $\boldsymbol{c}=[1,0,0,0,0,0,0,0]$.

One now readily sees how this mixer suppresses harmonics: the discrete Fourier transform of $\boldsymbol{a}$ has zeros at the $3^{\text {rd }}$ and $5^{\text {th }}$ harmonics, while that of $\boldsymbol{b}$ has zeros at all even harmonics. Thus, indeed the $2^{\text {nd }}$ through $6^{\text {th }}$ harmonic are suppressed.

In the full system of [5], four of these HRMs are combined into a complete quadrature-input and quadrature-output mixer.
Although it is easiest to simply treat these four mixers separately, the complete system can also be treated as a single full-quadrature mixer along the lines of section II-D.

\section{B. Zhiyu Ru et al.}

The two-stage HRM in [1], reproduced in Fig. 4b, enabled uncalibrated HR to better than $60 \mathrm{~dB}$. The first stage consists of three parallel low-noise transconductance amplifiers (LNTAs), with relative gains of 2,3 , and $2(\boldsymbol{a}=[2,3,2,0,0,0,0,0])$, feeding into three rotating switches with 8 positions $(n=8)$, operated by non-overlapping LO-signals. Of the resulting 8 output phases (buffered by the first transimpedance amplifiers (TIAs)), three adjacent-phase outputs are combined at the virtual ground of the second-stage TIAs via series resistors (i.e. with relative weights $1 / R$ ) to produce the $\mathrm{I}$ and $\mathrm{Q}$ outputs, thereby realizing only $y_{0}$ and $y_{2}$ in Fig. 2. The outputs of these second-stage TIAs are not inter-connected, so $\boldsymbol{c}=[1,0,0,0,0,0,0,0]$. The TIA feedback $R C$-networks create low-pass filters with an impulse response that can be modeled by $g(\cdot)$.

For $\boldsymbol{b}$, two different possibilities are given in [1]. The first, $\boldsymbol{b}^{\prime}=[5,7,5,0,-5,-7,-5,0]$, is analyzed theoretically by manually computing the effective mixing waveform to give $77 \mathrm{~dB}$ suppression of the third harmonic [1]. They basically compute the convolution described in (5) but without recognizing it as such. Using the insights of the present paper, we first compute the $3^{\text {rd }}$ harmonic attenuation of $\boldsymbol{a}$ as $30.6 \mathrm{~dB}$, and that of $\boldsymbol{b}^{\prime}$ as $45.9 \mathrm{~dB}$, which indeed adds up to $77 \mathrm{~dB}$.

When [1] turns to the practical implementation, $\boldsymbol{b}^{\prime}$ is actually replaced by $\boldsymbol{b}^{\prime \prime}=\left[\frac{1}{7}, \frac{1}{5}+\frac{1}{7}, \frac{1}{5}+\frac{1}{7}, \frac{1}{7},-\frac{1}{7},-\frac{1}{5}-\frac{1}{7},-\frac{1}{5}-\right.$ $\left.\frac{1}{7},-\frac{1}{7}\right]$. This replacement can be seen as the result of a circular convolution of $\boldsymbol{b}^{\prime}$ with $\frac{1}{35} \cdot[1,1,0,0,0,0,0,0]$. The discrete Fourier transform of $[1,1,0,0,0,0,0,0]$ has a rejection of the $3^{\text {rd }}$ harmonic of $7.7 \mathrm{~dB}$, which is added to the circuit's total HR. The zero-order hold in our model (see Fig. 3) contributes another $1.9 \mathrm{~dB}$ to the rejection of the $3^{\text {rd }}$ harmonic. These two improvements, yielding almost $10 \mathrm{~dB}$ extra suppression, have gone unnoticed in [1], as phase errors of the LO dominated 
the overall measured HR of $>60 \mathrm{~dB}$. This shows the strength of our approach, but also shows that an extension of the model to include effects like mismatch and gain/phase errors would be relevant as future work.

We immediately see that choosing $\boldsymbol{a}$ as $[5,7,5,0$, $-5,-7,-5,0]$ would have improved HR theoretically by another $15 \mathrm{~dB}$. This may seem a purely theoretical improvement, but would afford larger manufacturing tolerances.

\section{Travis Forbes et al.}

The 16-phase HRM presented in [7] also uses a two-stage approach, see Fig. 4c. By statically reconfiguring (permuting) the LO-to-switch pairing, the HRM can be used to receive one of the harmonics of the fundamental $\frac{f_{\mathrm{LO}}}{16}$ (where $f_{\mathrm{LO}}$ is $1 / \tau$ in our model), while suppressing the other harmonics, potentially including the fundamental.

To receive the $1^{\text {st }}$ harmonic, $\boldsymbol{a}=[0,4,7,9,10,9,7,4,0$, $0, \ldots, 0], \boldsymbol{b}=[1,0,0,0,0,0,0,0,-1,0,0,0,0,0,0,0]$, and $\boldsymbol{c}=[13,12,9,5,0,-5,-9,-12,0,0, \ldots, 0]$. Clearly, $\boldsymbol{b}$ eliminates all even harmonics. From the Fourier transforms of $\boldsymbol{a}$ and $c$ it follows that all odd harmonics are suppressed at least $36 \mathrm{~dB}$ by $\boldsymbol{a}$ and $44 \mathrm{~dB}$ by $\boldsymbol{c}$, or in total by at least $80 \mathrm{~dB}$. The only unsuppressed harmonic (below the $16^{\text {th }}$ ) is the $15^{\text {th }}$, which still has $23.5 \mathrm{~dB}$ of $\mathrm{HR}$ due to the sinc-filtering.

In general, for the $k^{\text {th }}$ harmonic, coefficients in these vectors need to be permuted: $\boldsymbol{a}_{i}^{\prime}=\boldsymbol{a}_{k i \bmod 16}$, and similar for $\boldsymbol{b}^{\prime}$ and $c^{\prime}$. Taking reception at the $k=2^{\text {nd }}$ harmonic as an example (at $0.2 \mathrm{~dB}$ loss due to sinc-filtering), $\boldsymbol{a}^{\prime}=[0,7,10,7,0,-7, \ldots]$, $\boldsymbol{b}^{\prime}=[1,0,0,0,-1,0, \ldots]$ and $\boldsymbol{c}^{\prime}=[13,9,0,-9,0,0, \ldots]$. Relative to the second harmonic, most harmonics are perfectly suppressed by $\boldsymbol{b}^{\prime}$, except for the $6^{\text {th }}$ (at $45.9 \mathrm{~dB}$ by $\boldsymbol{a}^{\prime}$ plus $39.5 \mathrm{~dB}$ by $\left.\boldsymbol{c}^{\prime}\right)$ and $10^{\text {th }}(91.8 \mathrm{~dB})$, while the first unsuppressed harmonic is the $14^{\text {th }}$ (still $16.9 \mathrm{~dB}$ due to the sinc-filtering). For higher desired harmonics similar results are found. Although the measured $\mathrm{HR}$ of around $70 \mathrm{~dB}$ is impressive, it is again, like [1], significantly below the theoretical HR from our model.

\section{CONCLUSiONS}

The framework presented in this paper enables the modeling of the HR performance of complicated multi-stage HRMs as a relatively simple stage, modulated by an LO signal that consists of a periodic Dirac impulse filtered by a multi-stage time-discrete filter. This enables several important conclusions to be drawn almost trivially.

Concretely, the advantages of our modeling method for multi-stage HRMs are the following:

- It is no longer needed to tediously calculate the total effective LO waveform (as done in e.g. [1]) in order to study the total HR; instead, the per-stage HRs in $\mathrm{dB}$ can simply be added.

- Analyzing the influence of manufacturing tolerances is easier, as each stage can be analyzed independently.

- Design can be more systematic, e.g. by targeting different harmonics at different stages and making a well-informed choice of what (integer) ratios to use.

Future work could focus on systematic mismatch (sensitivity) analyses on the HR performance, perhaps building on top of our framework.

\section{REFERENCES}

[1] Z. Ru, N. A. Moseley, E. A. M. Klumperink, and B. Nauta, "Digitally enhanced software-defined radio receiver robust to out-of-band interference," IEEE J. Solid-State Circuits, vol. 44, no. 12, pp. 3359-3375, Dec 2009.

[2] C. Andrews and A. C. Molnar, "A passive mixer-first receiver with digitally controlled and widely tunable RF interface," IEEE J. SolidState Circuits, vol. 45, no. 12, pp. 2696-2708, 2010.

[3] E. A. M. Klumperink and A. C. Molnar, "Interference robust, flexible radio receivers in CMOS," IEEE RFIC Virtual J., Oct. 2014.

[4] B. Razavi, "The harmonic-rejection mixer [a circuit for all seasons]," IEEE Solid-State Circuits Magazine, vol. 10, no. 4, pp. 10-14, 2018.

[5] J. A. Weldon, J. C. Rudell, Li Lin, R. Sekhar Narayanaswami, M. Otsuka, S. Dedieu, Luns Tee, King-Chun Tsai, Cheol-Woong Lee, and P. R. Gray, "A $1.75 \mathrm{GHz}$ highly-integrated narrow-band CMOS transmitter with harmonic-rejection mixers," in Proc. IEEE Int. Solid-State Circuits Conf. - Dig. Tech. Papers (ISSCC), Feb 2001, pp. 160-161.

[6] A. A. Rafi and T. R. Viswanathan, "Harmonic rejection mixing techniques using clock-gating," IEEE J. Solid-State Circuits, vol. 48, no. 8, pp. 1862-1874, Aug. 2013.

[7] T. Forbes, W. Ho, and R. Gharpurey, "Design and analysis of harmonic rejection mixers with programmable LO frequency," IEEE J. Solid-State Circuits, vol. 48, no. 10, pp. 2363-2374, Oct. 2013.

[8] J. Olde Dubbelink, "FM stereo coder schematic," ca. 1988, unpublished.

[9] H. Cha, K. Kwon, J. Choi, H. Kim, and K. Lee, "A CMOS wideband RF front-end with mismatch calibrated harmonic rejection mixer for terrestrial digital TV tuner applications," IEEE Trans. Microw. Theory Techn., vol. 58, no. 8, pp. 2143-2151, Aug. 2010.

[10] Y. Xu, J. Zhu, and P. R. Kinget, "A blocker-tolerant RF front end with harmonic-rejecting $N$-path filter," IEEE J. Solid-State Circuits, vol. 53 , no. 2, pp. 327-339, Feb. 2018.

[11] M. Bouhamame, L. L. Coco, S. Amiot, and S. Toutain, "A $60 \mathrm{~dB}$ harmonic rejection mixer for digital terrestrial TV tuner," IEEE Trans. Circuits and Systems I, vol. 59, no. 3, pp. 471-478, 2012.

[12] D. Im, O. Lee, and I. Nam, "A TV receiver front-end with linearized LNA and current-summing harmonic rejection mixer," IEEE Trans. Circuits and Systems II, vol. 64, no. 3, pp. 269-273, 2017.

[13] I. u. Din, J. Wernehag, S. Andersson, S. Mattisson, and H. Sjöland, "Wideband SAW-less receiver front-end with harmonic rejection mixer in 65-nm CMOS," IEEE Trans. Circuits and Systems II, vol. 60, no. 5, pp. 242-246, 2013.

[14] D. Murphy, H. Darabi, and H. Xu, "A noise-cancelling receiver resilient to large harmonic blockers," IEEE J. Solid-State Circuits, vol. 50, no. 6, pp. 1336-1350, 2015. 